\title{
UIED
}

\section{METODOLOGÍA PARA LA OPTIMIZACIÓN DE LA BASE DE DATOS DE LÍNEAS LÍMITE DEL INSTITUTO GEOGRÁFICO NACIONAL}

\section{Methodology for the optimization of the base of information of limit lines of the Geographical National Institute}

\author{
José María Fábrega Golpe ${ }^{1}$, Francisco García Cepeda², Antonio María Luján Díaz ${ }^{3}$ \\ y José Miguel Rubio Iglesias ${ }^{3}$
}

Recibido 14/12/2006; aceptado el 18/03/2007

Resumen. Esta metodología se ha desarrollado en el marco de un proyecto que es el objeto del Convenio Específico de Colaboración entre el Instituto Geográfico Nacional y la Escuela de Topografía de la Universidad Politécnica de Madrid relativo a la investigación, desarrollo, formación y difusión de conocimientos en el campo de las tecnologías de la información geográfica (TIG) para la investigación y desarrollo de la tecnología y metodología adecuada para la optimización de la información de la Base de Datos de Líneas Límite de la Dirección General del Instituto Geográfico Nacional.

El fin fundamental del mismo es desarrollar una metodología para mejorar la precisión de la Base de Datos de Líneas Limite que tiene el Instituto Geográfico Nacional. La exigencia actual de calidad y seguridad en la descripción geométrica de las líneas límite obliga a optimizar dicha descripción mediante la aplicación de nuevas tecnologías no existentes en el momento del levantamiento, y al diseño de metodologías adecuadas que, minimizando los tiempos y costes de ejecución, consideren asimismo los distintos agentes que participan en España en la definición de las líneas límite.

Para desarrollar dicha metodología será necesario en primer lugar digitalizar la información de los cuadernos de campo y las actas de deslinde existentes en el Instituto Geográfico Nacional, para que sea un trabajo abordable desde las tecnologías actuales; posteriormente, volcar la información referente a las líneas límite sobre ortofotografías a escala 1:5.000, a partir de los datos de los cuadernos de campo digitalizados. Se propondrá un nuevo sistema de gestión, tratamiento y almacenamiento de las líneas límite, con información sobre su linaje (origen de datos, precisión), así como el formato de salida de las propias líneas límite.

Para controlar la calidad de la metodología propuesta, se deberá validar la misma mediante un estudio teórico de la medida de rendimientos y precisiones y su verificación mediante toma de datos en campo. Particularmente, se llevará a cabo dicha validación en un conjunto de 140 líneas límite de 36 municipios de la provincia de Ávila y Segovia (los comprendidos en las hojas 556 y 457 del Mapa Topográfico Nacional 1:50.000).

Una vez contrastada la metodología y efectuados los oportunos procesos de refinamiento, se redactarán las conclusiones de todo el proyecto, que englobarán las recomendaciones de trabajo y las precisiones resultantes, los rendimientos de los diferentes procesos y los costes que se generen mediante el empleo de la nueva metodología.

Palabras clave: Línea Límite, Bases de Datos, Precisión, GPS, Topografía, Ortofotografía 
Abstract. This paper introduces the development of a methodology for the optimisation of the municipal boundaries database of the Instituto Geográfico Nacional. This project has arisen as part of a collaboration agreement between the Instituto Geográfico Nacional and the Escuela de Topografia of the Universidad Politécnica de Madrid which seeks to promote research, development and training in Geographic Information Technologies.

Current quality requirements demand the use of new technologies to improve the accuracy of the geometrical description of municipal boundaries. These technologies didn't exist when the municipal boundaries were first drawn up. Besides, it is convenient to design an appropriate methodology that minimises both costs and time employed.

The two main steps in the process are: first, the conversion of all the available data (fixing boundary minutes and field survey notebooks) into digital format in order to make possible their integration in a CAD system; and second, the display and visual overlay of these digital data over an 1:5000 orthophotography of the study area, to identify the boundary monuments. A new system will be proposed to manage, process and storage municipal boundaries information, including its lineage; an output format for these data will be designed as well.

In addition, a quality control will be designed to audit this scheme using Data Analysis and Statistical Inference techniques. Moreover, GPS technology will be used to get some boundary monuments co-ordinates to check the results of the proposed methodology.

The complete scheme will be tested in a study area corresponding to Ávila and Segovia provinces comprising 140 boundary segments from 36 municipalities.

Key Words: Municipal boundary, Database, Accuracy, GPS, Surveying, Orthophotography.

\section{INTRODUCCIÓN}

El Instituto Geográfico Nacional (IGN) ostenta, entre otras funciones, la responsabilidad de la ejecución de las certificaciones, dictámenes, levantamientos y replanteo de líneas límite jurisdiccionales. Gran parte de los trabajos de delimitación de términos municipales fueron llevados a cabo por el Instituto Geográfico y Estadístico, luego llamado Instituto Geográfico y Catastral y actualmente conocido como Instituto Geográfico Nacional, durante la etapa de formación del Mapa Topográfico Nacional 1:50.000, desde finales del siglo XIX hasta mediados del siglo XX.

A la hora de la formación de sucesivos documentos y productos cartográficos no se reobservaron estas líneas, sino que se utilizaron los levantamientos disponibles ya representados en las minutas a escala 1:25.000 que sirvieron para la generación del Mapa Topográfico Nacional (MTN50).

Con dicha información también se constituyó en dicha institución, a mediados de los años 1980, una Base de Datos de Líneas Limite (BDLL), con el fin de mantener actualizada la información acerca de las líneas límite del territorio español para emplearlas en distintos trabajos.

Con el paso del tiempo, la desaparición de muchos mojones, que señalizaban sobre el terreno las líneas límite, y la antigüedad de los documentos existentes referentes a las mismas hacen necesarias y a la vez dificultan enormemente las tareas de recuperación y replanteo de dichas líneas límite.

\section{CONCEPTOS FUNDAMENTALES}

\subsection{Línea límite jurisdiccional}

Una línea límite jurisdiccional constituye el trazado geométrico que delimita y separa dos términos municipales, es decir, las áreas sometidas a la autoridad o jurisdicción de un ayuntamiento. Excepcionalmente, un término municipal puede estar delimitado por una única línea límite, si está englobado dentro de un solo municipio, o bien, lo que es más habitual, por varias, si colinda con más términos municipales.

Una línea límite es, como toda división administrativa, un concepto intangible, una realidad inexistente en el terreno, que es materializada con mojones, hitos de granito o rocas autóctonas. Estos elementos representan los vértices de la estructura geométrica de la línea, que en general consiste en una poligonal de ejes rectos que encadena mojones sucesivos, pero que, en ocasiones, también se corresponde con algún elemento geográfico como una linde de parcela, un eje de camino o de un río, etc.

Los mojones extremos de una línea límite se denominan mojones tres términos, por ser comunes a tres términos municipales; en algunos casos, es posible que converjan cuatro términos en un mismo punto, por lo que éste se llamará mojón cuatro términos. Los intermedios, al pertenecer tan sólo a una línea límite, se denominan mojones dos términos 0 , simplemente, mojones. 


\subsection{Deslinde jurisdiccional entre términos municipales.}

Se llama operación de deslinde entre dos términos municipales al proceso de naturaleza jurídico-administrativa que establece la línea límite jurisdiccional entre dos municipios, ratificado por los Ayuntamientos implicados. Estas actuaciones llevan consigo el levantamiento de un acta de deslinde correspondiente.

Un acta de deslinde es un documento en el que se describen de forma literal todos los mojones de la línea límite que son comunes a dos términos municipales, o en el caso de los extremos, a tres o cuatro municipios, asi como las características de la geometría de la línea límite entre cada dos mojones consecutivos.

Actualmente, la demarcación, deslinde y amojonamiento de los términos municipales dentro de una Comunidad Autónoma está regulada por la legislación vigente en cada Comunidad Autónoma y el Reglamento de Población y Demarcación de las Entidades Locales, aprobado por el Real Decreto 1690/86, de 11 de Julio (B. O. E. de 14 de Agosto).

En cuanto a la demarcación de límites entre distintas Comunidades Autónomas, en desarrollo de lo dispuesto en el artículo 50.3 de la Ley 7/1985 se promulgó el Real Decreto $3426 / 2000$, de 15 de diciembre (B. 0. E. de 29 de Diciembre), por el que se regula este tipo de procedimiento de deslindes de términos municipales.

\subsection{Operaciones topográficas en los deslindes jurisdiccionales.}

Una vez ratificado el trazado de la línea límite por las Comisiones representantes de los Ayuntamientos implicados, y plasmado en el acta de deslinde, se procedía a efectuar trabajos topográficos que permitieran posteriormente el futuro replanteo de la línea límite. Clásicamente, estas operaciones topográficas se realizaban a partir de poligonales observadas con brújula taquimétrica y estadía, que enlazaban los sucesivos mojones de la línea límite.

La brújula taquimétrica era un instrumento topográfico muy empleado para medir direcciones y ángulos, aprovechando la propiedad de la orientación de su aguja magnética respecto de la dirección del norte magnético. Los ángulos observados se denominan rumbos magnéticos, siendo necesario conocer la declinación magnética existente en aquel momento en el lugar del levantamiento. Las ventajas de su sencillo y rápido manejo contrastan con la baja precisión de las observaciones realizadas con ella, debida fundamentalmente a las variaciones periódicas del magnetismo terrestre así como las anomalías puntuales debidas a las características del terreno.

Las observaciones realizadas mediante brújula y estadía quedaban anotadas en los denominados cuadernos de cam- po: rumbos, ángulos verticales, lecturas de mira, así como la declinación magnética en el momento de la observación e indicaciones acerca de la metodología llevada a cabo en la medición. Por lo tanto, en los cuadernos de campo quedaba definida geométricamente la línea límite de acuerdo a las disposiciones en el acta, aunque hay que tener en cuenta que los itinerarios que se describen en el mismo son libres analíticamente, es decir, que no se ajustan a puntos con coordenadas conocidas y precisas. Por lo tanto, no es posible con sólo estos datos referenciar de forma absoluta los puntos de la línea límite.

\subsection{Base de Datos de Líneas Límite (BDLL) del Instituto Geográfico Nacional.}

La Base de Datos de Líneas Límite se define como una Base de Datos que recoge la descripción geométrica y topológica de la división administrativa española, a los niveles de comunidad autónoma, provincia y municipio. Esta Base de Datos está disponible a diferentes escalas: 1:25.000, $1: 200.000$ y $1: 1.000 .000$, si bien los datos que la componen proceden de la digitalización de minutas de escala 1:25.000 que sirvieron para la creación del Mapa Topográfico Nacional 1:50.000, contrastados a su vez con actas, cuadernos de campo y croquis de los deslindes jurisdiccionales archivados en el IGN.

\section{OBJETIVOS}

El desarrollo de la metodología propuesta se basa en el cumplimiento de una serie de objetivos que se detallan a continuación.

\subsection{Objetivos generales}

- Elaborar una metodología de tratamiento de información adecuada que mejore la precisión geométrica de la Base de Datos de Líneas Límite existente en el Instituto Geográfico Nacional.

- La nueva metodología debe intentar sustituir en lo posible las tareas realizadas en campo por trabajo de gabinete, lo que supone una reducción de costes y tiempos así como una mayor productividad.

- La propuesta ha de contemplar la generación de un nuevo sistema de gestión y almacenamiento de líneas límite. Asimismo, el modelo de datos de líneas límite planteado tiene que atender a una estructura tramonodo coherente, consistente y completa.

\subsection{Objetivos específicos}

Para la consecución de los objetivos generales señalados, se plantean los siguientes objetivos específicos: 
- El fundamento del nuevo diseño se basará en el empleo de ortofotografías digitales como referencia para la representación de la geometría de las líneas límite.

- Para el desarrollo de la metodología se consultará la documentación y la cartografía existente en el Instituto Geográfico Nacional, principalmente actas de deslinde y cuadernos de campo, recurriendo asimismo a la experiencia del personal vinculado a las actividades relacionadas con el ámbito de las líneas límite.

- Los diseños metodológicos y tecnologías desarrolladas se deberán aplicar en una muestra previamente elegida para evaluar precisiones y rendimientos.

- Para alcanzar un mayor rendimiento tanto económico como productivo se implementará el mayor número posible de automatismos en las aplicaciones informáticas que se generen en el proyecto.

\section{DESARROLLO DE LA METODOLOGÍA DE OPTIMIZACIÓN DE LA BASE DE DATOS DE LÍNEAS LIIMITE.}

El desarrollo de este proyecto se estructuró en varias etapas, que a continuación se proceden a describir:

\subsection{Definición de las fases del proyecto $\mathrm{y}$ de las zonas de verificación de metodología.}

En un primer lugar se concretaron las fases de las que constaría este trabajo para cumplir los objetivos citados, que se detallaron en un documento o plan de trabajo. Asimismo se decidió, de forma conjunta con el Instituto Geográfico Nacional, el entorno geográfico en el que se debía aplicar la metodología propuesta en este proyecto para su ulterior verificación. Las zonas elegidas se correspondian con las líneas límite de las hojas 556 y 457 del Mapa Topográfico Nacional 1:50.000, en la provincia de Ávila y Segovia respectivamente. Las razones por las cuales estas líneas fueron las seleccionadas son:

- El conjunto de líneas límite seleccionado, tanto por orografía como por sus propias caracteristicas geométricas, es muy adecuado para implementar una metodología genérica.

- La proximidad a Madrid de las líneas seleccionadas conlleva una mayor flexibilidad en el trabajo, pues se pueden llevar a cabo las tareas de verificación y comprobación de la metodología de una manera más rápida y económica, teniendo en cuenta la relativa cercanía a las sedes del Instituto Geográfico y la Escuela de Topografía de la Universidad Politécnica.

\subsection{Descripción de los trabajos de deslinde llevados a cabo por el Instituto Geográfico Nacional.}

Dado que en los objetivos generales de este proyecto se aborda el desarrollo de una nueva metodología que optimice la BDLL y que esta metodología propone una alternativa al proceso actual de trabajo, se ha considerado de interés documentar las labores relacionadas con las líneas límite actualmente realizadas en el Instituto Geográfico Nacional. Con una descripción detallada se pretendía hacer un estudio comparativo entre los procesos actuales y la metodología propuesta para poder evaluar las ventajas tanto en sencillez del proceso y automatización, como en rendimiento y precisión.

Los trabajos de documentación e investigación se realizaron en todo momento con la cooperación del personal del Servicio de Deslindes y Grandes Escalas del Instituto Geográfico Nacional. El estudio se ha estructurado en los siguientes temas fundamentales relacionados con las líneas limite jurisdiccionales:

- Introducción acerca de los conceptos fundamentales relacionados con las líneas límite jurisdiccionales. Revisión histórica de los procedimientos de deslinde y marco jurídico actual.

- Exposición de la instrumentación y metodologías clásicas de levantamientos topográficos de líneas límite. Estudio de la brújula taquimétrica y los métodos de trabajo con la misma. Técnicas de medida indirecta de distancias. Recopilación de reglamentos para la ejecución de los trabajos de deslinde y amojonamiento llevados a cabo por el IGN.

- Descripción de los trabajos de recuperación y replanteo de líneas límite, llevados a cabo actualmente por el personal del Instituto Geográfico Nacional. Metodología y herramientas empleadas, resultados y documentación generada.

- Descripción de la formación y características de la Base de Datos de Líneas Límite (BDLL). Exposición de las tareas de la actualización de su geometría llevadas a cabo por el personal del Instituto Geográfico Nacional.

- Estudio de la documentación utilizada en las tareas descritas. Definición de acta de deslinde, cuaderno de campo, planimetría, ortofotografía así como otros documentos. Análisis de la aplicación empleada en el Instituto Geográfico Nacional para la visualización de actas y cuadernos.

\subsection{Desarrollo de la propuesta inicial de metodología}

Una vez analizado el estado actual de los trabajos relacionados con las líneas límite jurisdiccionales practicados en 
el Instituto Geográfico Nacional, se desarrolló la propuesta inicial de una metodología que de forma teórica permitiera alcanzar los objetivos de precisión y calidad planteados.

\subsubsection{Aspectos básicos de la metodología propuesta.}

La siguiente propuesta de trabajo presenta una metodología que consiste en desarrollar los itinerarios topográficos de líneas límite analítica y gráficamente en un entorno $C A D^{* 1}$ empleando como referencia ortofotografías digitales de escala 1:5.000. Mediante las observaciones anotadas en los cuadernos de campo se puede reconstruir matemáticamente los itinerarios levantados durante el deslinde jurisdiccional de cada línea límite. Además, si se desarrollan gráficamente sobre ortofotografías actuales, es posible advertir y corregir los errores que se acumulan en los ejes de los itinerarios derivados de la imprecisión de la metodología de observación topográfica empleada.

Por otro lado, estos itinerarios tienen que ajustarse a puntos de coordenadas precisas en el sistema de referencia de trabajo, ED50*2, con coordenadas en proyección $\mathrm{UTM}^{* 3}$ huso 30 (en el caso de las hojas de mapa de control). Dado que en los cuadernos de campo no existen referencias a puntos de coordenadas conocidas, en esta metodología se propone extraer coordenadas de puntos de las líneas límite reconocidos de forma univoca sobre la ortofotografía a escala 1:5.000.

Este procedimiento debe ser automatizado en el mayor número posible de fases que sea posible, con el fin de optimizar los tiempos de trabajo, mediante herramientas y aplicaciones creadas a tal efecto.

Finalmente, se propone la creación de un modelo de datos que se implementará en una base de datos relacional, almacenando digitalmente toda la información de los cuadernos de campo, los cálculos y las coordenadas de los puntos obtenidas, $y$, lo que es muy importante, las precisiones obtenidas en los cálculos efectuados para los puntos de las líneas límite.

\subsubsection{Documentación esencial empleada en la metodología.}

En esta metodología se proponen las siguientes fuentes de información en las cuales fundamentar los desarrollos a realizar:

- Cuadernos de campo y actas de deslinde: En los cuadernos de campo, como se ha citado anteriormente, se encuentran las observaciones topográficas de los itinerarios de líneas límite. Constituye la documentación esencial para desarrollar matemáticamente los itinerarios de líneas límite. En cuanto al acta de deslinde, al ser el documento legal que valida las características geométricas de la línea límite y contener la descripción literal de los mojones, es también imprescindible para representar gráficamente la línea límite así como identificar puntos sobre la ortofotografía. Las actas de deslinde y los cuadernos fueron aportadas por el Instituto Geográfico Nacional en formato digital ráster JPEG y son gestionados con una aplicación creada por el Instituto Geográfico Nacional, denominada SIDDAE, que permite su visualización.

- Ortofotografías a escala 1:5.000: Las ortofotografias empleadas están georreferenciadas en el sistema de referencia ED50, con coordenadas en proyección UTM. Proceden del SIGPAC, el Sistema de Información Geográfica para la Identificación de Parcelas agrícolas, siendo la resolución de la imagen de 0,5 m.

- Planimetrías a escala 1:25.000 de los términos municipales: son documentos cartográficos realizados a mano, que representan elementos geográficos planimétricos existentes en un determinado término municipal. La cartografía se encuentra en un sistema de referencia arbitrario y su objeto era contribuir a la formación del Mapa Topográfico Nacional 1:50.000. A pesar del gran intervalo de tiempo transcurrido, estos documentos contienen información valiosa que puede servir para realizar las tareas de desarrollo de la metodología. Estas planimetrías digitalizadas han sido aportadas por el IGN en formato JPEG, sin georreferenciar, por lo que es necesario transformar geométricamente la imagen antes de emplearla.

- Como fuente de datos inicial también se empleará la geometría de la propia BDLL, la actual Base de Datos de Líneas Límite del Instituto Geográfico Nacional. Como se ha citado anteriormente, su precisión es equiparable a la de una cartografía a escala 1:25.000, y puede servir como aproximación inicial en el desarrollo matemático de los itinerarios de líneas límite. El IGN proporcionó la BDLL en formato DGN de MicroStation.

\subsubsection{Desarrollo de lineas límite previos a la propuesta de metodología.}

Para plantear una metodología adecuada, se desarrollaron inicialmente, en una hoja de cálculo de Microsoft Excel,

\footnotetext{
*1 Diseño Asistido por Ordenador

*2 Datum Europeo de 1950

*3 Pryección Universal Transversa de Mercator
} 
los itinerarios topográficos de líneas límite de las zonas elegidas en la primera etapa del proyecto (Ávila y Segovia), implementándose las funciones necesarias para obtener las coordenadas de los puntos de las líneas límite. En el cálculo del itinerario se emplearon como coordenadas de los puntos inicial y final de los itinerarios las correspondientes a los mojones tres términos de la BDLL actual. Posteriormente, a partir de las coordenadas resultantes se generaba la geometría en un archivo de diseño de CAD. Con la ortofotografía como referencia se buscaban puntos identificables de la línea límite, modificando con estos puntos los itinerarios inicialmente calculados para que se ajustaran matemáticamente a ellos.

Después de realizar numerosas pruebas y estudios, se llegó a las siguientes conclusiones que sirvieron para plantear adecuadamente la metodología:

- Las tareas de la realización de líneas límite son difícilmente automatizables, aunque es posible generar aplicaciones informáticas que resuelvan el cálculo de las mismas contemplando las estructuras topológicas más usuales.

- Los operadores que trabajen en el cálculo, edición y ajuste de las líneas límite deben tener conocimientos topográficos y de ajuste de observaciones.

- No se tienen coordenadas precisas de los mojones extremos de las líneas límite, ni tampoco es posible en muchos casos extraerlos de las ortofotografías. Esto es necesario tenerlo muy en cuenta porque la red de líneas límite debe conformar una estructura tramonodo cerrada y los mojones tres términos constituyen los nodos de esta red.

En este momento se decidió también el entorno de trabajo de los operadores. Tras el estudio de los entornos CAD de MicroStation (de Bentley Systems) y AutoCAD (de Autodesk) para analizar cuál de ellos se adaptaba mejor al trabajo de cálculo y representación de líneas límite. Se optó finalmente por MicroStation, por un lado, porque que es capaz de admitir como ficheros de referencia las ortofotografías georreferenciadas que se iban a manejar sin necesidad de realizar transformaciones previas; por otro lado porque es más habitual como entorno de trabajo para aplicaciones cartográficas tanto en el IGN como en la Escuela de Topografía.

\subsubsection{Generación de la estructura inicial de una nueva base de datos de líneas limite.}

Como consecuencia de los objetivos planteados, era necesario crear la estructura inicial de una base de datos des- criptiva que almacenase la relación de todas las líneas límite y mojones tres términos existentes. Para proceder a ello, se empleó una herramienta $\mathrm{SIG}^{* 4}$, concretamente GeoMedia (de Intergraph $\mathrm{Co}$.), mediante la cual, apoyándose en la BDLL, se generasen las tablas de la base de datos correspondientes a las líneas límite, a los mojones tres términos y a los municipios, planteando asimismo las relaciones espaciales entre ellas. En realidad, de las líneas límite se conoce todo (cuántas y cuáles son, los municipios que separan, los mojones tres términos en los que comienzan y terminan, las otras líneas con las que están conectadas); todo menos su posición y su geometría precisa.

La idea fundamental del método empleado se basa en aplicar análisis topológico y herencia de atributos. Con la herramienta anteriormente citada, las entidades líneas límite heredan el atributo código INE de los municipios que separan, y posteriormente, los mojones tres términos adquieren los códigos de las líneas que delimitan, estableciendo así la relación entre dichas entidades. La relación entre líneas límite y mojones viene definida en una tabla adicional que permite conocer qué mojones tres términos separan cada línea límite. En la tabla de mojones tres términos se almacenan sus coordenadas extraídas de la BDLL, que sirven como coordenadas aproximadas para el cálculo del itinerario correspondiente. Gracias a esta estructura en el proceso de producción propuesto no es necesario introducir la definición de cada línea límite sino que estará ya almacenada en la base de datos, con lo cual el operador sólo tendrá que elegir de las existentes aquélla que va a calcular.

\subsubsection{Descripción del flujo de trabajo de la metodología.}

Considerando los objetivos del proyecto y las conclusiones obtenidas del desarrollo de un conjunto de líneas límite, la propuesta de trabajo que se planteó en un primer momento constaba de las siguientes fases:

- Procesos previos a la ejecución del cálculo de una línea límite.

- Recopilación de la documentación existente, teniendo especial cuidado en la elección del cuaderno de campo y del acta de deslinde conveniente. EI IGN es depositario de todos los cuadernos de campo y actas levantados, pero no todos ellos son válidos, por lo que es necesario estudiar qué documentación es la que se debe emplear.

- Georreferenciación de planimetrías, que pueden servir como fichero de referencia para identificar puntos de líneas límite sobre la ortofotografía.

${ }^{*} 4$ Sistema de Información Geográfica 
- Desarrollo de itinerarios topográficos y ajuste a puntos identificados con precisión sobre la ortofotografía.

Empleando una aplicación desarrollada a medida de esta metodología sobre MicroStation, el operador va a poder calcular el itinerario topográfico de cada línea límite, introduciendo las observaciones del cuaderno de campo a la vez que se va desarrollando gráficamente sobre la ortofotografía de modo interactivo. Asimismo, se podrán modificar los resultados obtenidos para adaptarlos a la ortofotografía manualmente. Los pasos a seguir son, básicamente:

- En la aplicación citada se selecciona, mediante consulta a la base de datos creada, la línea límite que se va a desarrollar, colocando como fichero de referencia automáticamente las ortofotografías correspondientes a la zona, así como la geometría de la BDLL, que sirve como aproximación inicial para el desarrollo de la línea límite.

- El operador introduce en la aplicación las características de la metodología de observación que se empleó en el itinerario de la línea límite, en función de la cual la aplicación posteriormente requerirá unos u otros datos. Además, el usuario indicará el valor de la declinación magnética correspondiente a la época en la que se levantó topográficamente y que aparece en los cuadernos de campo, así como la convergencia de la cuadrícula UTM en la zona de la línea límite seleccionada.

- Un mojón tres términos de una línea límite puede ser punto inicial del itinerario de dicha línea, pero, a su vez, puede ser vértice final de otra línea que converja en ese punto. Por ello, el programa permite que el usuario elija desde qué mojón tres términos va a comenzar el desarrollo y cálculo del itinerario.

- La aplicación a continuación despliega un interfaz en el que el usuario introduce los datos correspondientes a las visuales efectuadas en el itinerario: rumbos $\mathrm{N}$ y $\mathrm{S}$, lectura sobre la estadía, ángulo vertical. El programa calcula con estos datos las coordenadas de las estaciones de la poligonal, partiendo de las coordenadas aproximadas del mojón tres términos inicial seleccionado en la ventana anterior. A la vez, se irá desarrollando gráficamente el itinerario en el archivo de diseño. Algunas de las estaciones del itinerario serán, a su vez, mojones de la línea límite, por lo que, según se calculan estos puntos, también se dibujará automáticamente la propia línea límite, como se muestra en la Figura 1.

- En la aplicación se han implementado procedimientos de detección de errores groseros. El programa resalta convenientemente las posibles equivocaciones existentes en la introducción de los datos y observaciones correspondientes a la línea límite.

- Según se desarrolla el cálculo y se representan el itinerario y la propia línea límite, el operador, junto con el acta de deslinde, el cuaderno de campo y la planimetría, tratará de identificar algún punto de la línea con cierta precisión sobre la ortofotografía. En caso de encontrar un pun-

FIGURA 1. Visualización sobre ortofotografía de una línea límite obtenida tras la aplicación de la metodología y su itinerario topográfico frente a la procedente de la BDLL.

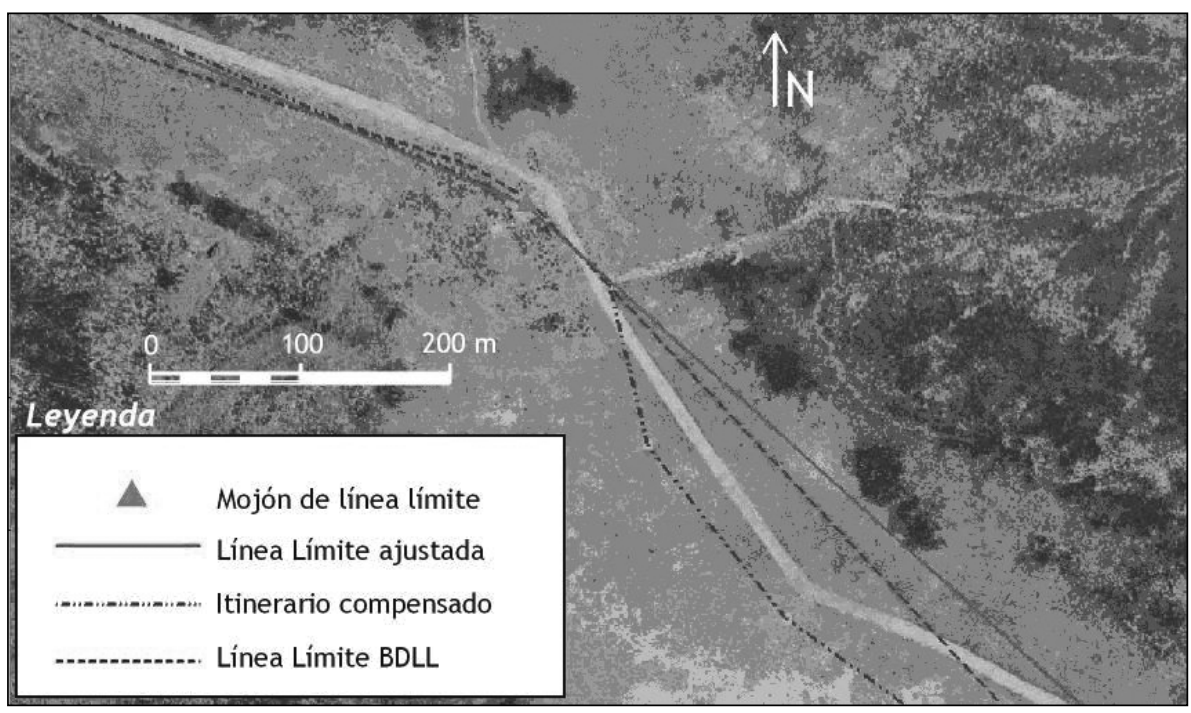

Fuente- Elaboración propia. 
to, con las coordenadas procedentes de la ortofotografía se recalculará el itinerario desarrollado hasta el mismo.

- Los tramos de itinerario que estén delimitados por puntos identificados de forma precisa sobre la ortofotografía, se ajustarán y compensarán una vez comprobado el error de cierre correspondiente. Los tramos que finalicen en mojones tres términos no identificados con precisión permanecerán sin compensar a la espera del cálculo de las líneas límite que cierran sobre dicho punto.

- En los itinerarios de líneas límite ya compensados se podrán modificar manualmente los tramos y mojones de la línea límite para que cumplan las especificaciones del acta de deslinde, sobre todo en aquellos casos en los que la línea discurra por algún elemento geográfico. Este procedimiento se efectuará entre cada dos puntos considerados como fijos, sin variar la posición de éstos, ya que es condición para la elección de los mismos que se hayan identificado de forma suficientemente precisa. Para poder realizar este ajuste gráfico será necesario en algunos casos introducir vértices en la línea, que sólo tendrán significado geométrico.

- La aplicación permite modificar las observaciones introducidas de líneas límite calculadas anteriormente, contemplando que en un futuro se pueda variar y mejorar las características de las mismas.

- Ajuste de las líneas límite a los mojones tres términos más probables.

Una de los requisitos fundamentales de la metodología es que esta genere un conjunto de líneas límite topológicamente correcto.

Dado el número de líneas límite existentes (del orden de 37.000) y su distribución geográfica (sobre una extensión de unos $500.000 \mathrm{~km}^{2}$ ), no se consideró posible un ajuste global del conjunto de líneas límite. Además, es muy importante, dado el volumen de trabajo total, ir teniendo conjuntos de municipios cerrados y ajustados, mientras se vayan calculando los demás.

Por tanto se diseñó un sistema mediante el cual a cada línea límite se le van dando coordenadas del sistema de referencia que se van considerando fijas, y luego se ajustan las líneas adyacentes para ir formando polígonos cerrados y ajustados.

Como se ha citado anteriormente, normalmente no será posible identificar de forma precisa los mojones tres términos de líneas límite en la ortofotografía. Por ello la aplicación calculará la localización más probable de un mojón tres términos a partir de las posiciones del mismo calculadas desde los distintos itinerarios que convergen, aplicando el criterio de dar mayor peso a las coordenadas procedentes de los tramos de menor longitud. Los itinerarios y, consecuentemente las líneas límite, se ajustarán a estos mojones tres términos más probables, generando así una red cerrada que se adapta al modelo de datos exigido. Posteriormente será posible editar manualmente los tramos que habian quedado sin adaptarse a las especificaciones del acta por no haber sido ajustados en ambos extremos.

- Almacenamiento, gestión y tratamiento de las líneas límite en una base de datos relacional.

Se ha creado un modelo de datos de líneas límite en el que se han definido las entidades principales con sus componentes y relaciones. En esta metodología, se proponen como entidades las provincias, los municipios, las líneas límite, los mojones tres términos y en general, los vértices que forman parte de la línea límite o de su itinerario. Estos vértices pueden ser:

- Mojones dos términos.

- Mojones tres términos.

- Vértices de la geometría de la línea límite, que se introducen para adaptar la línea a las disposiciones del acta de deslinde.

- Estaciones del itinerario, puntos que no tienen por qué pertenecer a la línea límite, pero son imprescindibles para su desarrollo matemático.

A estas entidades se les asocia una tabla en la nueva base de datos, en la que también se almacenarán las tablas correspondientes a las relaciones entre las entidades, tal y como se observa en la Figura 2. El motivo de crear dos tablas distintas para almacenar los vértices es el siguiente. Por un lado, almacenar en una tabla todas las observaciones de campo y conservarlas como información documental y, por lo tanto, la descripción original de la línea límite. En la otra tabla se almacenan las coordenadas de los vértices que se obtienen a lo largo del proceso de la metodología: identificación de vértices en la ortofoto, ajuste de las poligonales, etc.

En la etapa correspondiente a la creación de la estructura inicial ya se crearon algunas de estas tablas, rellenándose con datos de la provincia de Ávila y Segovia. Durante el proceso de cálculo efectuado con la aplicación, todas las observaciones de campo y las coordenadas de los puntos calculados, fijos y más probables, vértices de geometría y estaciones de itinerario así como las precisiones obtenidas tras el ajuste de los tramos serán almacenados convenientemente en los campos destinados a tal fin de las tablas de la Base de Datos. De esta manera, será posi- 
FiguRA 2. Modelo entidad-relación propuesto para la base de datos.

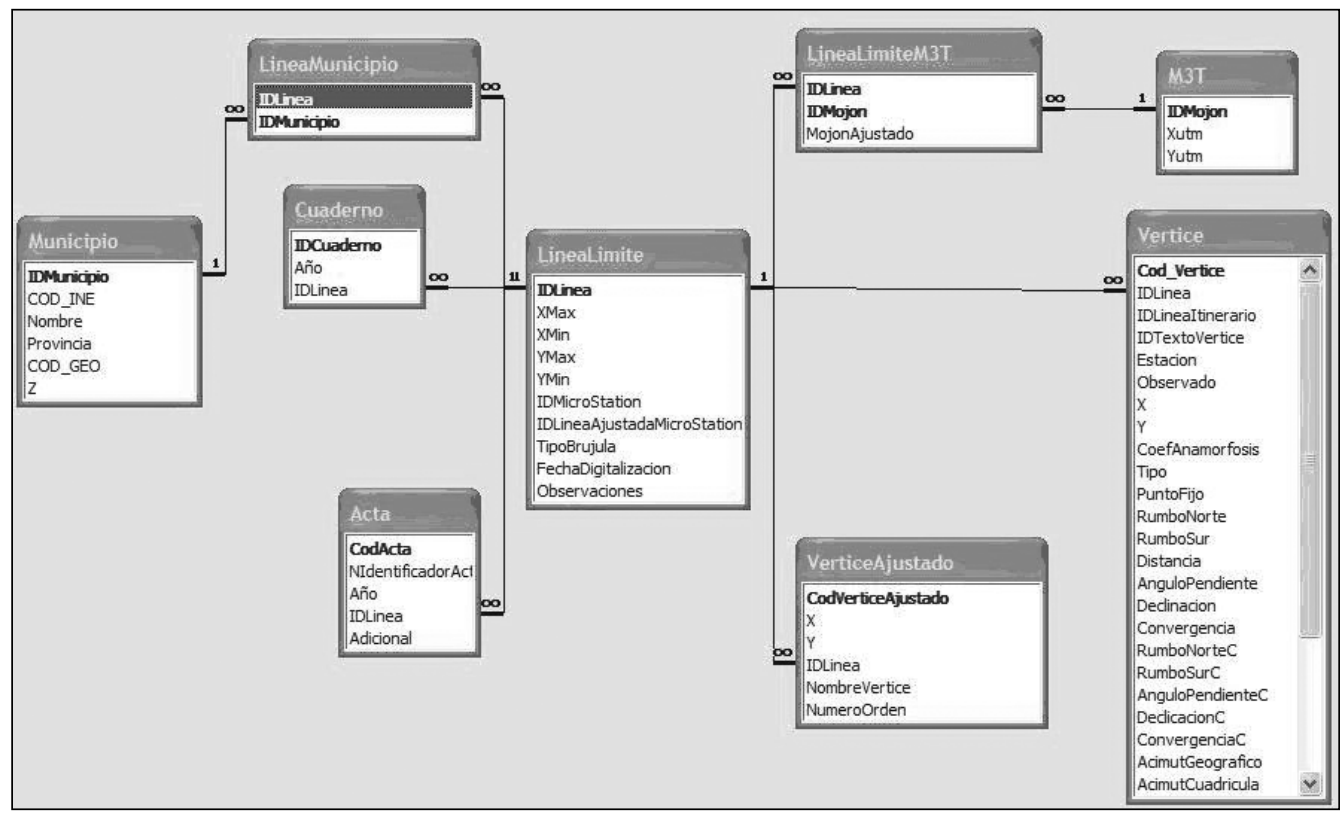

Fuente- Elaboración propia.

ble reconstruir las líneas límite en cualquier momento, ya que en las tablas quedarán completamente definidas.

\subsection{Control de calidad de la metodología}

Para evaluar las precisiones obtenidas en la aplicación de la propuesta metodologica se llevó a cabo un proceso de control de calidad dividido en las siguientes fases fundamentales: la etapa de toma de datos en campo con técnicas GPS, la correspondiente al análisis de resultados y, por último, la elaboración de conclusiones para plantear la metodología definitiva.

\subsubsection{Toma de datos en campo con instrumentación GPS}

Se realizó una toma de datos de un conjunto suficiente de líneas límite de las zonas de control anteriormente señaladas, que previamente se habian calculado y representado con ayuda de la citada aplicación. Para ello, se efectuó un estudio previo de las mismas, seleccionando mojones o puntos pertenecientes a la geometría de la línea así como aquellos puntos identificados en la ortofotografía con los que se habian ajustado y compensado los itinerarios. Con las coordenadas más o menos precisas de estos puntos, y con ayuda de un navegador se trató de localizar en campo el mayor número de ellos. Una vez identificados inequivocamente en el terreno, se observaron empleando la técnica GPS diferencial estático rápido. Estos datos fueron procesados en gabinete, obteniéndose coordenadas de estos puntos en el sistema de referencia de trabajo, que fueron incluidas en la aplicación para recalcular los itinerarios correspondientes, consiguiendo de esta manera una nueva representación de líneas límite.

\subsubsection{Análisis matemático de los resultados}

Con todos los datos disponibles, se realizó un completo estudio estadístico basado en análisis exploratorio de datos, contrastando los resultados de los diferentes desarrollos de líneas límite de la región de control mediante los siguientes análisis:

- Estudio analítico de las líneas límite de la BDLL existente en la actualidad frente a líneas que han sido calculadas con las observaciones de los cuadernos de campo. Estos itinerarios fueron desarrollados partiendo de las coordenadas extraídas de la BDLL de sus mojones tres términos iniciales y no fueron compensados ni mejorados en modo alguno. Por lo tanto, en este análisis se contrastaron estadisticamente los errores de cierre existentes en los mojones tres términos finales de cada línea límite frente su correspondiente representación en la BDLL. En este estudio de comparación mediante técnicas estadísticas, con datos agrupados y sin agrupar, se concluye que casi un 70\% de las líneas tienen un error de cierre por debajo de los $100 \mathrm{~m}$ pero algo más de la mitad presentan un error que supera los $50 \mathrm{~m}$.

- Estudio del cierre de líneas límite en los mojones tres términos más probables frente a los datos obtenidos en campo. Se realizó, en primer lugar, un estudio de 
la calidad del ajuste de los mojones tres términos obtenidos tras el cálculo de las líneas límite a partir de los puntos identificados en ortofotografía: se estudio la normalidad de los residuos del ajuste mediante el test de Kolmogorov-Smirnov; posteriormente, se calculó la calidad del ajuste a partir del test global del modelo y una combinación del test de Pope-Tau, que permite detectar la presencia de errores groseros en las observaciones, junto con el análisis de la configuración geométrica de cada uno de triángulos definidos por las soluciones de mojones tres términos provisionales de las líneas límite concurrentes. Posteriormente, se realizó el mismo proceso analítico con los mojones tres términos obtenidos a partir de la nueva representación de líneas límite obtenida a partir de la utilización de los datos observados en campo con GPS junto con puntos identificados en ortofotografía.

- Estudio comparativo de puntos pertenecientes a las líneas límite calculadas a partir de puntos fijos de la ortofotografía con sus coordenadas obtenidas en campo mediante observación GPS. Se analizaron las diferencias existentes, mediante un estudio de diversas medidas estadísticas de dispersión y de posición.

- Estudio comparativo de puntos pertenecientes a las líneas límite ajustadas y compensadas con puntos observados con GPS y puntos observados en ortofotografía frente a las coordenadas GPS de dichos puntos de control obtenidas en campo. El estudio analítico de las diferencias existentes arroja resultados que hacen posible esperar valores de precisión inferiores a 10 metros, pero que, sin embargo, indican la fuerte presencia de valores anómalos.

Paralelamente al estudio estadístico se analizó la distribución necesaria de puntos identificados en la ortofotografía a lo largo de una línea límite para alcanzar la precisión correspondiente a las diferentes escalas posibles de trabajo. De igual modo, se estudió la distribución de puntos que sería conveniente observar con tecnologías GPS en una línea límite para alcanzar determinadas precisiones en la representación de la misma. Con los resultados de este estudio, junto con el análisis estadístico anterior, se redactaron las conclusiones que se exponen en el siguiente apartado.

\subsubsection{Elaboración de conclusiones}

Con los análisis estadísticos efectuados, se desprendieron las siguientes conclusiones que sirvieron para la realización de una propuesta definitiva de optimización de la Base de Datos de Líneas Límite del IGN:

- Tras efectuar la comparación entre las coordenadas procedentes de la ortofotografía de puntos conside- rados como precisos en la metodología y las correspondientes obtenidas con instrumentación GPS, se determina que existe una imprecisión superior a la considerada originalmente en la elección de estos puntos. Ello es debido sobre todo a las características de los mojones de línea límite, ya que son difícilmente distinguibles en las imágenes disponibles, influyendo en ello tanto la propia resolución de la ortofotografía como el tiempo transcurrido que varía sustancialmente las descripciones de dichos puntos en el acta. También pueden ser fuente de error la calidad de la georreferenciación de la misma, las equivocaciones del propio operador en la identificación del punto y en la extracción de coordenadas así como un mal criterio en la elección sobre la ortofotografía de elementos que no son recomendables.

- Las características geomorfológicas del terreno y el trazado geométrico de las propias líneas límite son condicionantes importantes a la hora de estudiar la precisión alcanzable con la metodología inicial. Por un lado, terrenos muy homogéneos y poco contrastados, como berrocales o grandes extensiones de secano, imposibilitan en muchos casos la identificación correcta de puntos de control. Asimismo, líneas límite que no discurren por elementos del terreno y/o de gran longitud serian menos precisas que aquellas que se pudieran trazar sobre el eje de algún camino o río y/o con una reducida longitud.

- La necesidad del empleo de la documentación legal para la definición geométrica de las líneas actúa como factor limitante de la precisión que es posible alcanzar, siendo difícil cuantificar la exactitud de las descripciones literales. La metodología de observación topográfica llevada a cabo en el proceso de deslinde jurisdiccional, con el uso de brújula taquimétrica y estadía, supone también un condicionante insalvable. Del mismo modo, las características geométricas de los mojones y la virtualidad de las divisiones administrativas, provoca que sea complicado hablar en términos de precisión absoluta de los vértices que las conforman. Por último, una incidencia superior a la esperada de errores groseros en las anotaciones del cuaderno de campo, implica la necesidad de un tratamiento con mayor profundidad y un estudio caso a caso de los problemas encontrados.

- En definitiva, la definición de las líneas límite resultante de la aplicación de la nueva metodología mejora con mucho la precisión geométrica de las líneas procedentes de la BDLL, pero no alcanza la calidad exigida inicialmente debido a la imposibilidad de reconocer puntos de las líneas límite en la ortofotografía con la adecuada precisión por las causas ante- 
riormente expuestas y la existencia importante de errores groseros en las observaciones.

- Los resultados extraídos de la observación GPS son, por las técnicas empleadas, más precisos que los obtenidos con la metodología propuesta inicialmente, pero entre los objetivos de esta metodología se indicaba la necesidad de minimizar en lo posible el trabajo de campo para reducir costes y tiempos de trabajo. Además, en otros aspectos, no es viable una metodología basada exclusivamente en datos GPS, teniendo en cuenta que en muchos casos la línea límite discurre por elementos geográficos difícilmente accesibles o identificables en campo. Por otro lado, muchos de los mojones se han perdido, por lo que en estos casos no es posible identificarlos en campo.

- Una adecuada combinación de datos GPS y ortofotografía permitiría alcanzar el punto óptimo de precisión, rendimiento y costes. Los puntos calculados con observaciones con metodologías GPS, si es posible identificarlos correctamente, conllevan una holgada exactitud teniendo en cuenta que una línea limite tiene carácter administrativo, y como tal es inexistente en la naturaleza y materializada únicamente con hitos o mojones en muchos casos de grandes dimensiones. Por ello, la precisión que cabe esperar en el desarrollo de itinerarios con puntos GPS depende principalmente de la propagación de errores de observación a lo largo del itinerario. Por otro lado, caminos o arroyos, por cuyos ejes discurre la línea límite, en muchos casos se pueden identificar con precisión suficiente en la ortofotografía, de forma mucho más sencilla, rápida y precisa que con instrumentación GPS en campo. Ello implica, por lo tanto, que también pueden emplearse para alcanzar una correcta definición de la geometría de la línea. Asimismo, otros puntos identificados en ortofotografías con gran exactitud igualmente permitirian alcanzar los objetivos planteados.

\subsection{Propuesta definitiva de metodología}

Concluidas las labores de toma de datos en campo y verificación de los resultados obtenidos por los diferentes métodos planteados, se realizó la propuesta definitiva que contempla las siguientes modificaciones a la metodología inicial:

- La metodología definitiva incluye la observación de puntos con tecnologías GPS, debido a que la precisión que se puede alcanzar en el desarrollo de los itinerarios con estos datos satisface los objetivos de calidad exigidos.

- La metodología de observación GPS debe garantizar 3 metros de precisión en los puntos observados, sien- do conveniente emplear técnicas que reduzcan el tiempo de estacionamiento en cada punto para disminuir costes en el trabajo de campo.

- El aumento de coste que supone esta propuesta puede minimizarse estableciendo un número suficiente de puntos de observación GPS para garantizar las precisiones requeridas. Este número dependerá de las características de la metodología de observación del itinerario de la línea límite, así como de la morfología del terreno y del trazado y longitud de la línea. El número de puntos GPS necesario para mantener las precisiones exigidas en la representación geométrica de las líneas límite puede reducirse a su vez si la línea límite discurre por algún elemento geográfico perfectamente identificado en la ortofotografía, o bien si el operador es capaz de elegir en la imagen algún punto con una indeterminación inferior a 5 metros. En general, una buena opción para salvar precisiones consiste en tratar de identificar en campo con GPS los mojones tres términos o mojones cercanos a éstos, y, si se trata de una línea límite de gran longitud, algún mojón intermedio. Asi, varias líneas límite quedarian ajustadas en puntos con gran precisión, aislando los posibles errores existentes en tramos pertenecientes sólo a una línea límite.

- La aplicación tiene que permitir, por lo tanto, introducir puntos fijos con diferentes precisiones, es decir, diferenciando en el cálculo puntos GPS frente a puntos que se puedan distinguir en una ortofotografia, teniéndolo en cuenta a la hora de obtener las precisiones alcanzables en los itinerarios correspondientes.

Por lo tanto, conseguir una precisión propia de la escala de observación en la representación de la geometria de las líneas límite jurisdiccionales es una utopía tratándose de líneas administrativas materializadas en el terreno con piedras, hitos o mojones que superan dichas dimensiones en la mayoría de los casos, y observadas a su vez con metodologías que imposibilitan, por sus características intrínsecas, alcanzar dichas magnitudes.

Pero, en definitiva, se puede alcanzar una precisión geométrica del orden de 5 a $10 \mathrm{~m}$, considerablemente superior a la existente actualmente, combinando el desarrollo interactivo de líneas límite sobre ortofotografías digitales de alta resolución junto con el empleo de observaciones de campo efectuadas con instrumentación GPS.

\section{BIBLIOGRAFÍA}

ARIZA, F.J.: Calidad en la producción cartográfica. Madrid, Editorial RaMa. 2002

CASELLA, C. Y BERGER, R.L.: Statistical Inference. California, Duxbury Thomson Learning. 2002 
José María Fábrega Golpe, Francisco Garcia Cepeda, Antonio Maria Luján Diaz y José Miguel Rubio Iglesias

CHUECA PAZOS, M. y otros: Tratado de Topografía Tomo II. Métodos Topográficos. Madrid, Editorial Paraninfo. 1996

DOMinGUEZ GARCIAA-TEJERO, F.: Topografía General y Aplicada. Madrid, Editorial Dossat. 1965

GÓMEZ VILLEGAS, M. A.: Inferencia Estadistica. Madrid, Editorial Diaz de Santos.
SILVERMAN, B.W.: Density Estimation for Statistics and Data Analysis. Monographs on Statistics and Applied Probability 26. Boca Ratón, Florida, Chapman\&tHall/CRC. 1992

TUKEY, J.W. : Exploratory Data Analysis. E.E.U.U., Addison-Wesley Publishing Company. 1977 